\title{
Characterization of low-mass pre-main sequence stars in the Southern Cross ${ }^{\star}$
}

\author{
J. M. Alcalá ${ }^{\text {, E. Covino }}{ }^{1}$, C. Melo ${ }^{2}$, and M. F. Sterzik ${ }^{3}$ \\ 1 Osservatorio Astronomico di Capodimonte, Via Moiariello 16, 80131 Napoli, Italy \\ 2 Observatoire de Genève, Ch. des Maillettes 51, 1290 Sauverny, Switzerland \\ 3 European Southern Observatory, Casilla 19001, Santiago 19, Chile
}

Received 5 November 2001 / Accepted 9 January 2002

\begin{abstract}
We report high-resolution spectroscopic observations, as well as high-resolution near infrared (IR) imaging of six stars previously identified in a ROSAT pointed observation in the direction of the B-type star $\beta \mathrm{Cru}$, and classified as low-mass pre-main sequence (PMS) stars. Four of the stars are confirmed to be low-mass PMS stars, associated with the Lower Centaurus-Crux group, while the other two are unrelated to the Sco-Cen association. The confirmed PMS stars are most likely in their post-T Tauri evolutionary phase. Although future deep X-ray observations with high-resolution imagers might detect more new PMS stars, the possibility that the Crux PMS stars are part of a small aggregate, with $\beta$ Crux itself approximately at the center, is rather unlikely, given the high velocity dispersion and the low spatial density of the confirmed PMS stars. Instead, these stars may be part of a moving group in a more disperse and numerous population of low-mass PMS stars, distributed in the Lower Centaurus-Crux subgroup. New PMS binaries and multiple systems were also discovered among the stars in the sample, namely a close visual pair and a hierarchical triple system in which one of the components is a double-lined spectroscopic binary (SB2). The detailed orbital solution is reported for the inner short-period $\left(P_{\text {orb }}=58.3\right.$ days $)$ SB2. A preliminary orbital solution for the hierarchical triple system yields a systemic orbital period of about 4.6 years, which makes this object a very suitable target for follow-up observations with the Very-Large Telescope Interferometer (VLTI) in the coming years.
\end{abstract}

Key words. stars: pre-main sequence - stars: low mass, brown dwarfs - stars: binaries: general - X-rays: stars

\section{Introduction}

The discovery by the Einstein X-ray satellite that many low-mass pre-main sequence (PMS) stars are strong X-ray sources (Walter 1986), led many researchers to pursue the search for X-ray emitting low-mass pre-main sequence stars. A lot of progress in this field has been accomplished in the last decade by the observations of the ROSAT satellite which detected many new weak $\mathrm{T}$ Tauri stars (WTTS), not only in the star forming clouds, but also in the surroundings of star formation complexes (see Feigelson \& Montmerle 2000; Walter et al. 2000, and references therein).

The origin of the scattered population of WTTS has been somewhat debated. One of the main reasons for such

Send offprint requests to: J. M. Alcalá,

e-mail: jmae@na.astro.it

* Based on observations carried out at the European Southern Observatory, La Silla, Chile under proposals numbers 62.I-0368 and 63.I-0045, 65.I-0089 and the Swiss Euler telescope at ESO, La Silla. a debate has been the low spectroscopic resolution used for the identification of the WTTS candidates, because the presence of strong Lithium $\lambda 6707$ absorption cannot be unambiguously assessed. Some researchers argued that such objects are not PMS stars, but young zero-age mainsequence (ZAMS) stars (Briceño et al. 1997; Favata et al. 1997; Micela et al. 1993). However, several investigations, based on high-resolution spectroscopy, demonstrated that most of the widely scattered WTTS are indeed low-mass PMS stars (Covino et al. 1997; Wichmann et al. 1999; Alcalá et al. 2000). Feigelson (1996) suggested that the large number of scattered WTTS were formed in cloudlets which dissipated immediately after star formation. Other authors proposed that a considerable number of these stars may be members of the Gould Belt (Wichmann et al. 1997; Guillout et al. 1998).

The follow-up observations of the ROSAT discovered WTTS using high-resolution spectroscopy have also allowed the identification of a considerable number of PMS spectroscopic binaries among these stars. For instance, several double line spectroscopic binaries were 
discovered in the Chamaeleon (Covino et al. 1997), Lupus (Wichmann et al. 1999) and Orion (Alcalá et al. 2000) star forming regions (SFRs), which have increased significantly the number statistics of PMS spectroscopic binaries Melo et al. (2001). The PMS binaries are of crucial importance because the determination of dynamical masses, by the solution of their orbits, allows to put constraints on the theoretical PMS evolutionary tracks. Recently, Covino et al. (2001) have solved the orbits of some of these systems in Orion, and the first eclipsing PMS binary with solar-mass components was also discovered among that sample (Covino et al. 2000).

In a ROSAT pointed observation on the region of the B-type star $\beta$ Cru, Park \& Finley (1996, hereafter PF96) found a group of six X-ray emitting stars to be good candidates for WTTS. They suggested that these stars might be members of a previously unrecognized star forming region which includes $\beta$ Crux itself. Feigelson \& Lawson (1997, henceforth FL97) used low-resolution spectroscopy to sudy these objects and concluded that they are PMS stars, although not forming part of a previously unrecognized star forming region, but being members of the low-mass PMS population of the Sco-Cen association, and representing just a few of the many WTTS in Sco-Cen.

In this paper, we characterize the Crux stars of the PF96 sample by means of high-resolution spectroscopic observations and near infrared imaging. In Sect. 2, we present the observations and data reduction. In Sect. 3, we describe the determination of radial and rotational velocities, as well as of the effective temperatures and line equivalent widths. In Sect. 4, we report the discovery of two binaries in the sample, namely a close visual binary, and a double-lined spectroscopic binary (SB2), whose orbital solution is also presented. Finally, we discuss the PMS nature of the stars in Sect. 5.

\section{Observations and data reduction}

\subsection{Spectroscopy}

The high-resolution spectroscopic observations were performed using three instruments at the European Southern Observatory (ESO), La Silla, Chile: the Cassegrain Echelle Spectrograph (CASPEC) attached to the ESO $3.6 \mathrm{~m}$ telescope, the Fiber-fed Extended Range Optical Spectrograph (FEROS) attached to the ESO $1.5 \mathrm{~m}$ telescope and CORALIE at the Euler $1.2 \mathrm{~m}$ Swiss telescope.

The first set of observations was obtained using CASPEC on February 1999. The CASPEC data reduction was performed using the Echelle reduction package available within the Munich Image Data Analysis System (MIDAS, version November 1997), plus some specially devised procedures making use of the algorithms prescribed by Verschueren \& Hensberge (1990) for background subtraction and optimal order extraction. The nominal resolving power of these spectra, as measured from several isolated lines of the thorium-argon comparison spectrum, is $\lambda / \Delta \lambda \approx 22000$. Unfortunately, CASPEC was very close to be decommissioned during our observing period and many technical problems related to the positioning of the cross-disperser prevented us from performing a reliable wavelength calibration of the spectra to derive radial velocities. Therefore, additional spectra for all the stars were obtained with FEROS in May 1999 and January 2001.

Despite of the problems with the cross-disperser, the CASPEC spectra allowed us to detect the Lithium resonance line at $6708 \AA$, wherever present, and to reveal a new double-lined spectroscopic binary (SB2). Systematic observations of this SB2 were immediately started with CORALIE, and since April 1999 also using FEROS (see Sect. 4.2).

The reduction of the FEROS data was performed using the specific FEROS data-reduction software (DRS) implemented in the ESO-MIDAS environment (from MIDAS version $98 \mathrm{NOV}$ on). The basic reduction consisted of the following steps: i) definition of the echelle orders on flatfield frames; ii) background subtraction; iii) extraction of the echelle orders; iv) flat-fielding of the extracted spectra (to remove pixel-to-pixel variations as well as correct for the blaze function); v) wavelength calibration using ThAr exposures; vi) rebinning to wavelength scale; vii) merging of the orders. For details on the instrument and on the data reduction procedures we refer to the FEROS User's Manual (Francois 1999, Vers. 1.1) and The FEROS Cookbook (Pompei \& Francois 2000, Vers. 2.2), respectively ${ }^{1}$.

The nominal resolving power of the FEROS spectra, as measured from several isolated lines of the thorium-argon comparison spectrum, is $\lambda / \Delta \lambda \approx 48000$.

For the CORALIE spectra with a resolution of 47000 all observations were taken with one fiber centered on the target star and the other fiber illuminated by the background sky. The reduction is performed by an on-line reduction procedure: after reading the CCD, the spectrum is extracted, calibrated in wavelength and flat-fielded. The on-line reduction system also performs the crosscorrelation of the stellar spectrum with a numerical mask (Queloz 1995) for the determination of radial and rotational velocities.

In Table 1 a summary of the spectroscopic observations is presented. The number of observations of Cru3 reported in this table refer only to the indicated periods. The sample of observations of Cru-3 are reported in Sect. 4.2 , in Table 4.

In Fig. 1, CASPEC and FEROS spectra of the sample stars in the range from $\mathrm{H} \alpha$ to the Lithium $\lambda 6707 \AA$ absorption line are shown.

As one can see from Fig. 1, with the exception of Cru5 , all the other stars show the Li I $\lambda 6708$ absorption line. FL97 give an upper limit of $0.2 \AA$ for the Li equivalent width of Cru-5, but their spectrum for this star has a low $S / N$ ratio. Unfortunately, FL97 do not specify which of the stars in the X-ray error boxes reported by PF96 they

\footnotetext{
1 http://www.ls.eso.org/lasilla/Telescopes/2p2T/ E1p5M/ FER0S/docu/Ferosdocu.html
} 
Table 1. Summary of spectroscopic observations.

\begin{tabular}{lccc}
\hline Star & CASPEC & FEROS & No. \\
\hline \hline Cru-1 & 5-Feb.-99 & 17-May-99; 09-Jan.-01 & 3 \\
Cru-2E & 5-Feb.-99 & 09-Jan.-01 & 2 \\
Cru-2W & - & 17-May-99 & 1 \\
Cru-3 $^{\dagger}$ & 5-Feb.-99 & - & 1 \\
Cru-4 & 6-Feb.-99 & 19-May-99; 04-Jan.-01 & 3 \\
Cru-5 & 6-Feb.-99 & 20-May-99 & 2 \\
Cru-6 & 6-Feb.-99 & 09-Jan.-01 & 2 \\
\hline
\end{tabular}

$\dagger$ See Table 4 for FEROS and CORALIE observation dates.
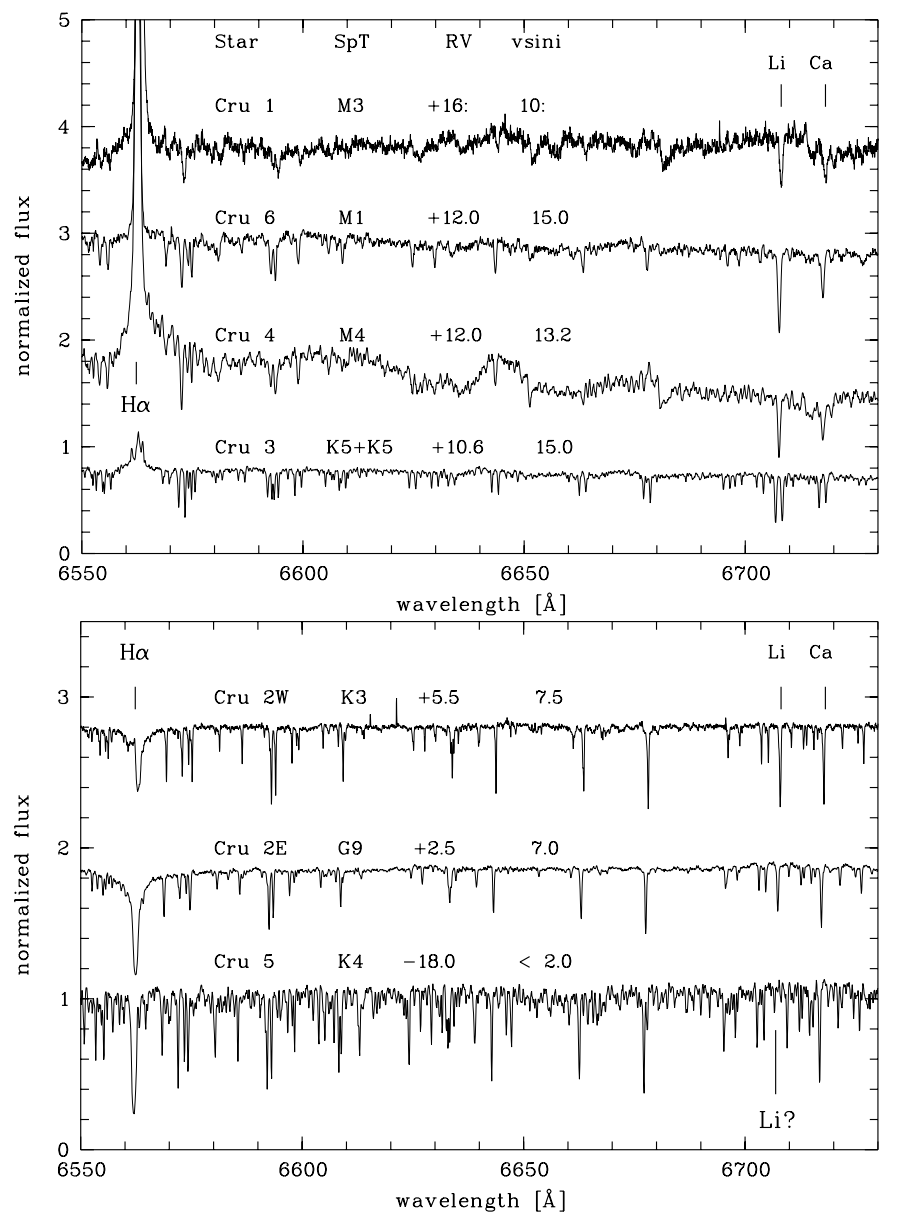

Fig. 1. High-resolution CASPEC and FEROS spectra of the stars in our sample in the $\mathrm{H} \alpha$ - Lithium range. Note the double spectral lines of the star Cru-3. The spectra of the stars Cru-2E, $3,4,5$ and 6 are from CASPEC, while those of Cru- 1 and Cru$2 \mathrm{~W}$ are from FEROS. The spectral type (SpT), radial velocity $(\mathrm{RV})$, and projected rotational velocity (vsini) are indicated for each star. For Cru-3 the systemic radial velocity is reported (see Sect. 4.2 for details). The ":" in the values of $\mathrm{Cru}-1$ indicate variability (see Sect. 4.1.)

have observed. Therefore, unless we have observed a different object (which we consider rather unlikely because the spectral type we assign to this star is consistent with the one reported by FL97), we do not find any Li I absorption in Cru-5. As already reported by FL97, the stars Cru-1, Cru-3, Cru-4 and Cru-6 have $\mathrm{H} \alpha$ in emission, while
Table 2. Near-infrared photometry, radial and projected rotational velocities of the Crux stars.

\begin{tabular}{lrrrrr}
\hline Star & $J$ & $J-H$ & $H-K$ & $\begin{array}{r}R V \\
{\left[\mathrm{~km} \mathrm{~s}^{-1}\right]}\end{array}$ & $\begin{array}{r}v \sin i \\
{\left[\mathrm{~km} \mathrm{~s}^{-1}\right]}\end{array}$ \\
\hline \hline Cru-1 & 10.28 & 0.69 & 0.22 & $+16.0:$ & $10.0:$ \\
Cru-2E & 9.04 & 0.35 & 0.09 & +2.5 & 7.0 \\
Cru-2W & 9.05 & 0.47 & 0.12 & +5.5 & 7.5 \\
Cru-3 $^{\dagger}$ & 8.23 & 0.68 & 0.15 & +10.6 & 15.0 \\
Cru-4 $_{\text {Cru-5 }}^{10.06}$ & 0.70 & 0.20 & +12.0 & 13.2 \\
Cru-6 & 10.56 & 0.70 & 0.19 & -18.0 & $<2.0$ \\
\hline
\end{tabular}

$\dagger$ The systemic $R V$ is given. See also Table 4 for FEROS and CORALIE measurements.The ":" means variable radial and rotational velocity

the couple Cru-2E and Cru-2W and the star Cru-5 show $\mathrm{H} \alpha$ in absorption.

\subsection{Near infrared imaging}

Near infrared $J, H, K$, imaging of the six Crux stars was performed at ESO, La Silla, with the ESO $3.6 \mathrm{~m}$ telescope and the ADaptive Optics Near Infrared System (ADONIS) using the SharpII camera on January 11, 2001. A pixel scale of $0.05 \mathrm{arcsec} / \mathrm{pixel}$ was selected, and we used an internal chopping method for sky subtraction. A detector integration time (DIT) of $5 \mathrm{~s}$ for all sample stars and all three filters was chosen, except for Cru-3 (DIT = $2 \mathrm{~s}$ ). Ten independent frames have been acquired in each filter for all objects except for Cru-1, for which we obtained 20 images per filter. The adaptive optics loop was closed on the science target itself for all the objects. The airmass during the observations varied between 1.19 and 1.26. Standard processing techniques within the ECLIPSE software $^{2}$ was applied for flat-fielding, dark correction, bad pixel removal, and shift and add. From the IR standard stars AS16-2 and AS16-4 (Hunt et al. 1998) we determined the photometric zero points and the mean photometric errors. Table 2 summarizes the results for the six stars.

Atmospheric extinction coefficients in the three bands were determined using the observations of the comparison star in the field of the PMS eclipsing binary RXJ 0529.4+0041, observed during several hours on the same night and spanning an air-mass from 1.0 to 1.8 (see ESO-press release $22 / 01^{3}$; Covino et al., in preparation).

The mean zero points are $22.34 \pm 0.01,22.06 \pm 0.02$ and $21.51 \pm 0.03$ in the $J, H$ and $K$ bands respectively. The mean photometric errors are $\sigma_{J}=0.04, \sigma_{H}=0.04$ and $\sigma_{K}=0.06$. The zero points are in very good agreement with those reported by the ESO $3.6 \mathrm{~m}$ telescope team. More details on the data reduction will be reported in Covino et al. (in preparation).

\footnotetext{
2 http://www.ls.eso.org/lasilla/Telescopes/360cat/ adonis/ html/datared.html\#eclipse

3 http://www.eso.org/outreach/press-rel/pr-2001/ pr-22-01.html
} 
The star Cru-1 resulted to be a close visual pair with a separation of $0.25 \operatorname{arcsec}$ (see Sect. 4.1), while the star Cru-2, previously known to be a visual binary, has a separation of 3.25 arcsec. The latter is thus sufficiently well separated to allow aperture photometry of the individual components.

When comparing the IR colours of the Crux stars with those of normal field stars and IRAS sources in star forming regions, it is found that the Crux stars lack IR excesses (cf. Fig. 2): while the stars Cru-2E and $2 \mathrm{~W}$ have near-IR colours consistent with those of normal field stars, the other Crux stars fall in an intermediate region between the IRAS sources and the normal field stars, although they tend to follow the line of normal colours for field dwarfs, indicating the lack of near-IR flux excesses. The different position of Cru-2E and $2 \mathrm{~W}$ in the $J-H$ versus $H-K$ diagram compared to the other Crux stars is mainly due to the earlier spectral type of Cru- $2 \mathrm{E}$ and $2 \mathrm{~W}$.

Since the components of the binary Cru-3 are practically equal, the IR colours are the same for both components. On the other hand, it was possible to resolve the visual binary Cru- 1 only in the $K$ band therefore, we could not determine the colours of the individual components.

\section{Parameter determinations}

\subsection{Radial velocity and $v \sin i$}

Due to the serious problems with the cross-disperser positioning during the February 1999 observations, no radial velocity determinations were possible from the CASPEC spectra.

Determinations of radial velocity, $R V$, and projected rotational velocity, $v \sin i$, were obtained using instead the FEROS spectra, and in the case of the SB2 Cru-3 also the CORALIE data, by means of cross-correlation analysis. Given the large spectral coverage achievable with FEROS and CORALIE, the cross-correlation of the target and template spectra was performed after rebinning the spectra to a logarithmic wavelength scale, in order to eliminate the dependence of Doppler shift on the wavelength (Simkin 1974). Moreover, only parts of the spectra free of emission lines and/or not affected by telluric absorption lines were considered. Therefore, the NaI D, and $\mathrm{H} \alpha$ lines as well as wavelengths longer than about $7000 \AA$ have been excluded from the cross-correlation analysis. The result of the cross-correlation is a correlation peak which can be fitted with a Gaussian curve. The parameters of the Gaussian, center position and fullwidth at half-maximum $(F W H M)$ are directly related to $R V$ and $v \sin i$, respectively. The method of the correlation has been fully described by Queloz (1995), and Soderblom et al. (1989). More details about the calibration procedure can be found in Appendix A of Covino et al. (1997). The measured $R V$ and $v \sin i$ determinations for the program stars are reported in Fig. 1 and Table 2. The typical measured errors are of the order of $1 \mathrm{~km} \mathrm{~s}^{-1}$ and $1.5 \mathrm{~km} \mathrm{~s}^{-1}$ for $R V$ and $v \sin i$ respectively.

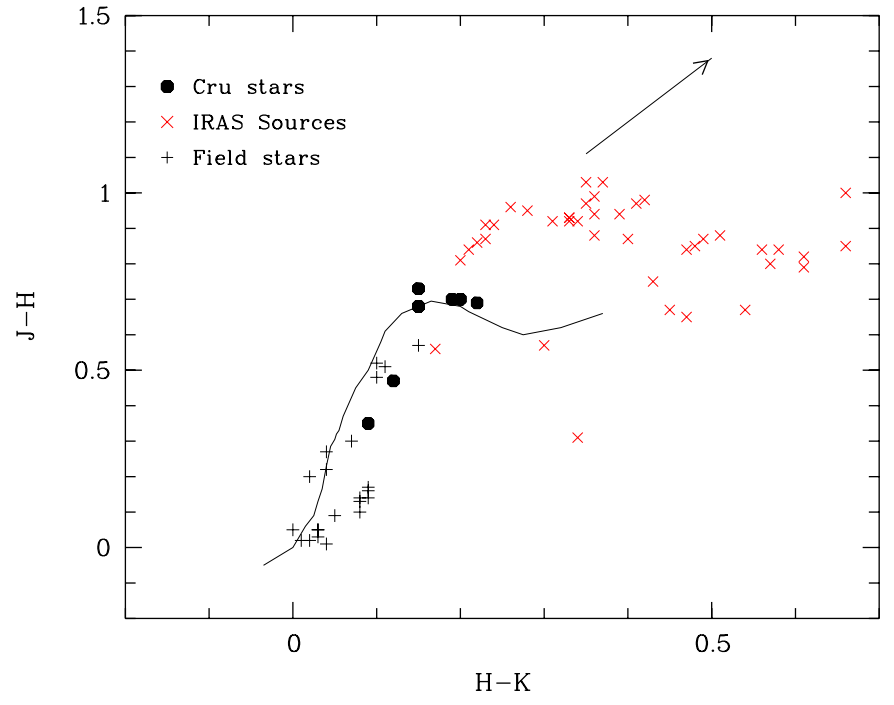

Fig. 2. Comparison of the IR colours of the Crux stars (dots) with those of normal field stars $(+)$ and IRAS sources $(x)$ in star forming regions. The solid line represents the colours of normal field dwarfs from Bessel \& Brett (1988). The arrow indicates the normal reddening vector.

\subsection{Spectral types and effective temperatures}

Exploiting the large spectral range covered by the CASPEC and FEROS spectra, we could assign spectral types to the target stars following the procedure described in Covino et al. (1997) and Alcalá et al. (2000).

For the stars earlier than K7, an estimate of the effective temperature has been performed using the calibrations between the Na I D lines equivalent width and $\log T_{\text {eff }}$ for luminosity class $\mathrm{V}$ given by Tripicchio et al. (1997), while for cooler stars the relationship between the K I $\lambda 7699$ equivalent width and $\log T_{\text {eff }}$ for luminosity class V, reported in Tripicchio et al. (1999), was used.

The derived effective temperatures are consistent, within the errors, with those derived using the calibration between spectral type and effective temperatures (e.g. de Jager \& Nieuwenhuijzen 1987). The spectral type and effective temperature for the Crux stars are reported in Table 3.

\section{3. $H \alpha$ and $L i$ equivalent widths and Li abundances}

$\mathrm{H} \alpha$ and Li I $6708 \AA$ line equivalent widths were determined from the normalized CASPEC and FEROS spectra by integration of the line, interpolating linearly the continuum on both sides of the line.

The main source of error on these measurements comes from the uncertainty in the placement of the photospheric continuum. For each spectrum, at least three individual measurements of $W(\mathrm{H} \alpha)$ and $W(\mathrm{Li})$ were obtained by setting the continuum at different positions. The mean estimated error of $W(\mathrm{Li})$ is $10 \mathrm{~m} \AA$ in most cases, while for $W(\mathrm{H} \alpha)$ the error is about $10 \%$. For the stars later than M1, in which the continuum placement is difficult 
Table 3. Derived parameters for the stars in the Crux sample.

\begin{tabular}{lccrcccc}
\hline Crux & SpT & $\begin{array}{c}\log T_{\text {eff }} \\
{[\mathrm{K}]}\end{array}$ & $\begin{array}{r}W_{\mathrm{H} \alpha} \\
{[\AA]}\end{array}$ & $\begin{array}{r}W_{\mathrm{Li}} \\
{[\mathrm{m \AA}]}\end{array}$ & $N_{\mathrm{Li}}$ & $A_{\mathrm{V}}$ & $\begin{array}{r}\log L \\
{\left[L_{\odot}\right]}\end{array}$ \\
\hline \hline 1 & $\mathrm{M} 3$ & 3.532 & -6.50 & 0.395 & 1.04 & 0.13 & -0.85 \\
$2 \mathrm{E}$ & $\mathrm{G} 9$ & 3.719 & 1.94 & 0.150 & 2.60 & 0.00 & -0.14 \\
2W & $\mathrm{K} 3$ & 3.671 & 0.94 & 0.230 & 2.33 & 0.00 & -0.22 \\
$3 \mathrm{a}$ & $\mathrm{K} 5$ & 3.644 & -0.48 & 0.460 & 2.82 & 0.63 & -0.10 \\
3b & $\mathrm{K} 5$ & 3.644 & -0.52 & 0.480 & 2.89 & 0.63 & -0.10 \\
4 & $\mathrm{M} 4$ & 3.517 & -5.80 & 0.420 & 1.17 & 0.07 & -0.92 \\
5 & $\mathrm{~K} 4$ & 3.657 & 1.00 & - & - & 1.25 & -0.67 \\
6 & $\mathrm{M} 1$ & 3.564 & -3.20 & 0.570 & 2.14 & 0.10 & -0.60 \\
\hline
\end{tabular}

because of photospheric absorption bands, the uncertainty of $W(\mathrm{Li})$ may be as high as $25 \mathrm{~m} \AA$.

Lithium abundances, in the usual scale $\log (H)=12$, were derived from the $W(\mathrm{Li})$ and $T_{\text {eff }}$ values using the non-LTE curves of growth given by Pavlenko \& Magazzù (1996), assuming $\log g=4.5$.

The main source of error on the derived $\log N(\mathrm{Li})$ values is the uncertainty in the effective temperature. The estimated mean uncertainties on $T_{\text {eff }}$ are on the order of $\Delta T_{\text {eff }} \approx 150 \mathrm{~K}$. Taking this and a mean error of about $15 \mathrm{~m} \AA$ in $W(\mathrm{Li})$ into account, we estimate a mean error on the order of 0.15 to 0.2 dex in $\log N(\mathrm{Li})$. However, the assumption of $\log g=4.5$ affects significantly the lithium abundance determination, in the sense that a lower surface gravity yields a higher lithium abundance. In particular, for stars with $\log T_{\text {eff }}$ less than about $3.7(\approx 5000 \mathrm{~K})$ and $\log W(\mathrm{Li})$ greater than about $2.5(\approx 320 \mathrm{~m} \AA)$, the difference in $\log N(\mathrm{Li})$ may rise to 0.3 dex, when assuming $\log g=3.5$. Hence, assuming $\log g=3.5$ would result in higher lithium abundances than when assuming $\log g=4.5$. Thus we adopt the most conservative value, $\log g=4.5$, which might eventually lead to an underestimation of the abundance.

In the case of the spectroscopic binary Cru-3, we used the method reported in Covino et al. (2001) in order to determine the weighting factors and correct for the contribution of each binary component to the observed total continuum. Since the two components are quite similar, the weighting factor is practically 0.5 for each of them.

The $\mathrm{H} \alpha$ and lithium equivalent widths $W(\mathrm{Li})$, as well as the lithium abundances are reported in Table 3 . We adopt the convention that positive equivalent widths indicate absorption lines.

By comparison with the values reported in Table 1 of FL97, we notice that the strength of the $\mathrm{H} \alpha$ emission line of Cru-1, Cru- 3 and Cru- 6 is quite variable, as it is expected in active, young stars.

\subsection{Bolometric luminosities}

The methods described in Alcalá et al. (1997) were used to calculate the bolometric luminosities, assuming that the six Crux stars are located at the same distance as the B0.5IV type star $\beta$ Cru, i.e. 110 pc (Perryman et al. 1997). A normal interstellar extinction law was assumed

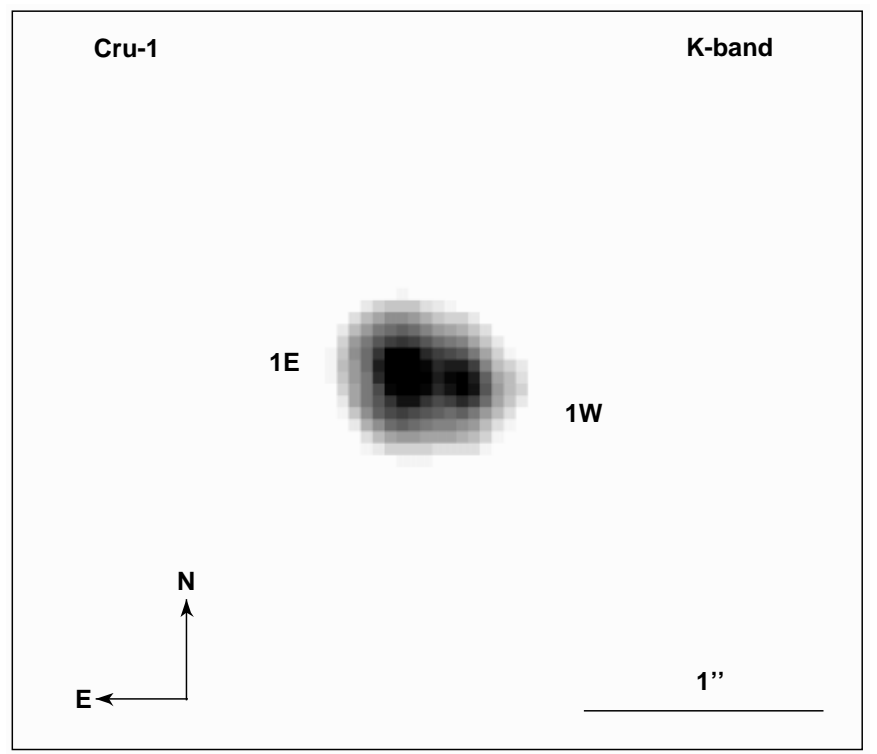

Fig. 3. The visual binary Cru- 1 in the $K$ band. The image scale is 50 mas/pix. The separation of the components is about 0.25 arcsec and the position angle is about $76^{\circ}$. The flux ratio of the components in this band is about 1.8.

in order to derive the intrinsic colours and reddening. The interstellar extinction, $A_{\mathrm{V}}$, and the stellar luminosities are reported in Table 3.

The stellar luminosities calculated in this way are overestimated for the binary stars. For equal binary components, one can derive the individual luminosities simply by subtracting $\log 2$ to the total luminosity. The luminosities derived in this way for the components of Cru-3 are reported in Table 3 ; in the case of $\mathrm{Cru}-1$, it is more difficult to estimate the individual luminosities, because there is no information on the individual spectral types or colours. As a first approximation, one can assume that the luminosity ratio of the components is the same as the flux ratio measured in the $K$ band (see Sects. 2.2 and 4.1), and hence subtract $\log (1+1 / 1.8)$ and $\log (1+1.8)$ to the logarithmic total luminosity, for the primary and secondary components respectively.

\section{New PMS binaries}

\subsection{The visual binary Cru-1}

The near-IR imaging shows that the star Cru-1 is a close visual pair. Unfortunately, the binary is only marginally resolved in the $J$ and $H$ bands, while it can just be resolved in the $K$ band with a separation of about 0.25 arcsec and a position angle of about $76^{\circ}$ (cf. Fig. 3). The flux ratio of the components in the $K$ band is about 1.8, the East component being brighter than the West component.

Some evidence of a variable radial velocity was found from the cross-correlation analysis. Therefore, one cannot exclude that Cru-1 may also be a spectroscopic binary. 


\subsection{The spectroscopic binary Cru-3}

The double-lined spectroscopic binary nature of Cru-3 was revealed in the course of our observing run with CASPEC in February 1999. Since then, the system was systematically observed during several observing runs conducted with CORALIE and FEROS, on La Silla (Chile). The observations were performed in different epochs during 1999, 2000 and 2001. The radial velocities of the system were determined applying cross-correlation techniques as explained in Sect. 3.1. All the radial velocity measurements for Cru-3 are listed in Table 4.

A first, preliminary orbital solution for Cru-3 was obtained early in June 1999, using all FEROS and CORALIE data available at that moment. The solution of the spectroscopic orbit was obtained using standard non-linear least squares techniques (e.g., Press et al. 1992) on all data points, except those where the two components were seen in blend. From this, the following orbital elements were determined: the orbital period, $P_{\text {orb }}$, the radial velocity of the center of mass, $\gamma$, the semi-amplitudes of the radial velocity curves of each component, $K_{1}$ and $K_{2}$, the eccentricity, $e$, the longitude of periastron, $\omega$, and the time of periastron passage, $T$. Other derived quantities include the projected semi-major axes, $a_{1} \sin i$ and $a_{2} \sin i$, the minimum masses of the components, $M_{1} \sin ^{3} i$ and $M_{2} \sin ^{3} i$, and, of course, the mass ratio, $q$.

Since, by that time, only half of the radial velocity curve was satisfactorily covered by the observations, we continued collecting data in order to achieve a better coverage of the entire curve but, surprisingly, the dispersion around the orbital solution was found to increase continuously with the addition of new data. We also noticed, however, that the radial velocities observed for both components in January 2001 with FEROS appeared shifted some $10 \mathrm{~km} \mathrm{~s}^{-1}$ with respect to the first orbital solution obtained in 1999, although the relative radial velocity between the two components was in good agreement with the predictions from the former orbital solution. Such a shift in the radial velocity of both components strongly suggests that the barycentric velocity of the binary system is changing due to the presence of a third body and therefore, any attempt to fit new and old data sets simultaneously, while keeping the $\gamma$ parameter fixed, failed.

Hence, we adopted a different approach in order to find out whether a barycentric velocity variation was really occurring in this system. We chose the observations obtained with CORALIE during May 1999 as a reference, since it was in this run that a longer series of consecutive observations were collected, allowing a good orbital solution with the data of this run alone. The orbital solution found by using only the CORALIE data of May 1999 is hereafter referred to as the reference solution. Then we imposed the orbital parameters from the reference solution for the other blocks of observations obtained in other epochs allowing only the barycentric velocity to vary freely. As shown in Fig. 4, a marked trend in the $\gamma$ velocity (varying from about 15 down to about $3 \mathrm{~km} \mathrm{~s}^{-1}$ ) is present,
Table 4. Radial velocity measurements, in $\mathrm{km} \mathrm{s}^{-1}$, for the SB2 components of Cru-3.

\begin{tabular}{|c|c|c|c|}
\hline HJD-2400000 & $R V_{a}$ & $R V_{b}$ & Instr. \\
\hline 51216.765407 & $\overline{48.210}$ & -20.370 & $\overline{\text { CORALIE }}$ \\
\hline 51253.768142 & -8.092 & 38.534 & CORALIE \\
\hline 51260.784077 & 10.555 & 19.182 & CORALIE \\
\hline 51274.686200 & 48.000 & -21.500 & FEROS \\
\hline 51275.683360 & 47.500 & -21.000 & FEROS \\
\hline 51311.568220 & -9.050 & 39.480 & CORALIE \\
\hline 51312.735649 & -6.530 & 36.930 & CORALIE \\
\hline 51313.731979 & -4.080 & 34.570 & CORALIE \\
\hline 51314.735635 & -1.530 & 32.120 & CORALIE \\
\hline 51315.176678 & 0.400 & 28.400 & CORALIE \\
\hline 51315.670575 & 0.680 & 29.410 & CORALIE \\
\hline 51315.680420 & 1.000 & 29.500 & FEROS \\
\hline 51316.216678 & 3.400 & 25.200 & CORALIE \\
\hline 51316.580283 & 3.020 & 26.830 & CORALIE \\
\hline 51316.720410 & 3.500 & 27.500 & FEROS \\
\hline 51317.226979 & 7.200 & 21.400 & CORALIE \\
\hline 51317.662823 & 6.210 & 23.600 & CORALIE \\
\hline 51317.730690 & 6.500 & 24.000 & FEROS \\
\hline 51318.144826 & 14.000 & 14.000 & CORALIE \\
\hline 51318.634322 & 8.910 & 20.540 & CORALIE \\
\hline 51318.648520 & 15.000 & 15.000 & FEROS \\
\hline 51319.180359 & 14.000 & 14.000 & CORALIE \\
\hline 51319.684030 & 15.000 & 15.000 & FEROS \\
\hline 51320.647870 & 15.000 & 15.000 & FEROS \\
\hline 51321.146088 & 14.800 & 14.800 & CORALIE \\
\hline 51321.616683 & 14.720 & 14.720 & CORALIE \\
\hline 51321.649730 & 15.000 & 15.000 & FEROS \\
\hline 51328.678780 & 41.350 & -10.110 & CORALIE \\
\hline 51364.500093 & -15.520 & 46.090 & CORALIE \\
\hline 51365.506992 & -14.860 & 45.340 & CORALIE \\
\hline 51366.500928 & -13.850 & 44.400 & CORALIE \\
\hline 51368.489771 & -11.250 & 41.810 & CORALIE \\
\hline 51526.853957 & 1.190 & 26.790 & CORALIE \\
\hline 51527.825863 & -1.410 & 30.770 & CORALIE \\
\hline 51528.801809 & -4.200 & 33.590 & CORALIE \\
\hline 51529.841162 & -6.780 & 36.270 & CORALIE \\
\hline 51530.852991 & -9.270 & 38.610 & CORALIE \\
\hline 51533.833932 & -14.320 & 43.740 & CORALIE \\
\hline 51667.717920 & 5.700 & 20.300 & FEROS \\
\hline 51672.591324 & 20.210 & 2.190 & CORALIE \\
\hline 51674.726350 & 27.800 & -4.400 & FEROS \\
\hline 51682.717875 & 44.550 & -23.810 & CORALIE \\
\hline 51684.647100 & 45.200 & -23.700 & FEROS \\
\hline 51686.662950 & 44.500 & -22.500 & FEROS \\
\hline 51687.617595 & 43.090 & -22.730 & CORALIE \\
\hline 51918.849790 & 35.500 & -33.000 & FEROS \\
\hline 51923.850610 & 29.000 & -25.000 & FEROS \\
\hline 52019.604800 & 4.500 & 4.500 & FEROS \\
\hline 52026.612060 & 26.000 & -19.000 & FEROS \\
\hline 52031.598260 & 37.000 & -29.200 & FEROS \\
\hline
\end{tabular}

confirming our suspicious of a changing barycentric velocity and hence the presence of a third body.

The final orbital solution, obtained combining all available data points from both FEROS and CORALIE, was found as follows: for a given run, $R_{i}$, with CORALIE (FEROS), the observed radial velocities, $V_{\mathrm{r}, \mathrm{obs}}$, were 


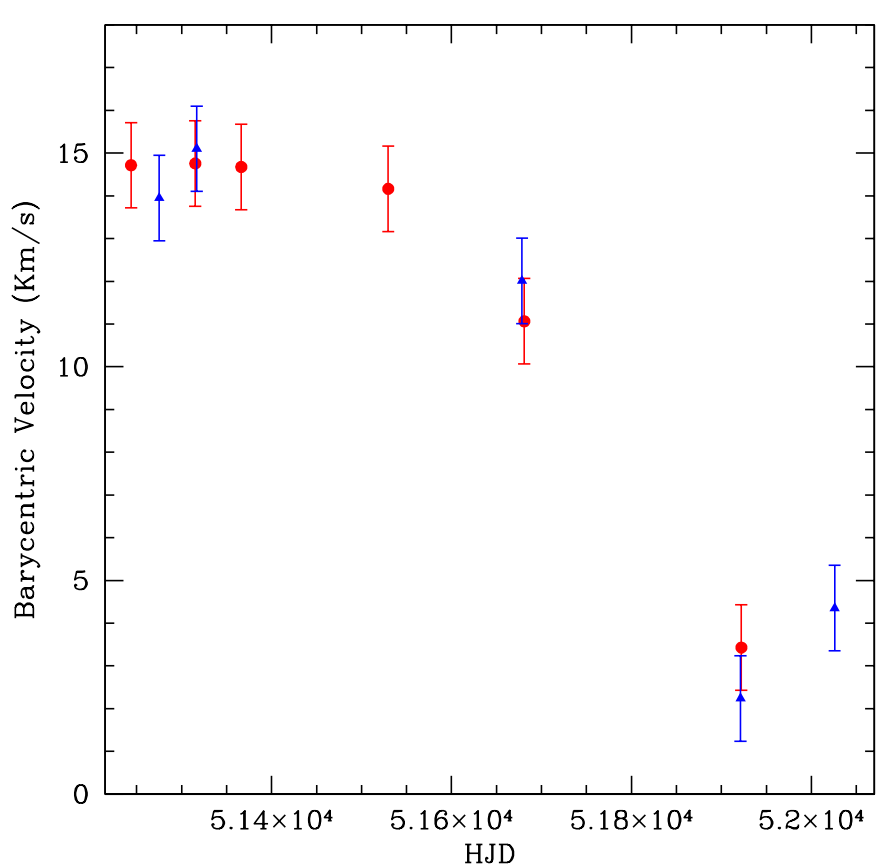

Fig. 4. Barycentric velocity, $\gamma$, of the SB2 system Cru-3 as a function of time. Each data point corresponds to the average barycentric velocity derived for each observing run versus the mean Heliocentric Julian Date for that run. Circles and triangles represent CORALIE and FEROS runs, respectively.

corrected by a constant $k_{i}$ such as: $V_{\mathrm{r}, \mathrm{cor}}=V_{\mathrm{r}, \mathrm{obs}}+k_{i}$, where $k_{i}=\gamma_{\text {May99 }}-\gamma_{i}$, with $\gamma_{\text {May99 }}$ the barycentric velocity of the CORALIE (FEROS) orbital solution obtained with the data of May 1999 and $\gamma_{i}$ the barycentric velocity derived from the data obtained in the considered run. At this point, the radial velocity data collected with each of the two instruments are reported to the same reference frame of May 1999, using the $\gamma$ values reported in Table 5. A final correction still remains to be made, namely, tie the FEROS data to the reference frame of CORALIE. This is done by adding another constant $k^{\prime}$ to the already corrected (as above) FEROS data, where $k^{\prime}=\gamma_{\text {May/99,CORALIE }}-\gamma_{\text {May99,FEROs. }}$. The final orbital solution was then found by using all corrected data with all orbital parameters allowed to vary. Figure 5 shows the corrected radial velocity curve of Cru-3 SB2 components and the corresponding best fit, whereas Fig. 4 shows the systemic radial velocity, $\gamma$, as a function of time. The results of the orbital solution are reported in Table 6 . As one can see from the spectrum shown in Fig. 1, the components of this double-lined spectroscopic binary are very similar and, in fact, from the orbital solution it turns out that the mass ratio is about 0.95 .

Figures 4 and 5 clearly show that Cru-3 is in fact a hierarchical triple system, i.e., a long period binary system in which one of the components is itself a binary. From Fig. 4, one can clearly see that the spectroscopic binary, Cru$3 \mathrm{AB}$, has not yet completed an entire orbital revolution around the center of mass of the system Cru-3AB+Cru$3 \mathrm{C}$. However, since the value of the barycentric velocity from the most recent FEROS observations of April 2001

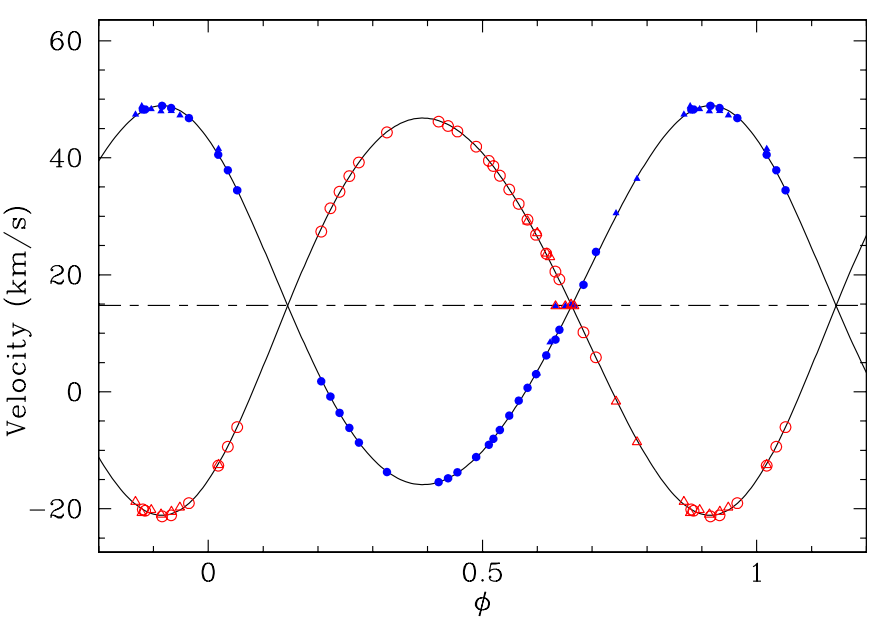

Fig. 5. Radial velocity curve of Cru-3. The data points for the primary and secondary components are represented by filled and open symbols, respectively, and the corresponding orbital fit, as solid lines. The circles and triangles represent the CORALIE and FEROS points respectively.

Table 5. Barycentric velocity, $\gamma$, for each CORALIE and FEROS observing runs. $<$ HJD $>$ corresponds to the mean Heliocentric Julian Date $(-2400000)$ for each run. The barycentric velocity is given in $\mathrm{km} \mathrm{s}^{-1}$.

\begin{tabular}{lcr}
\hline Run & $\langle$ HJD $\rangle$ & $\gamma$ \\
\hline & CORALIE \\
\hline Feb./99 & 51243.7725 & 14.715 \\
May/99 & 51315.1649 & 14.757 \\
Aug./99 & 51366.2494 & 14.675 \\
Dec./99 & 51529.6682 & 14.164 \\
May/00 & 51680.9756 & 11.064 \\
Jan./01 & 51922.2441 & 3.429 \\
\hline & FEROS \\
\hline Apr./99 & 51275.1848 & 13.949 \\
May/99 & 51316.7105 & 15.101 \\
May/00 & 51678.4386 & 12.009 \\
Jan./01 & 51921.3502 & 2.241 \\
Apr./01 & 52025.9384 & 4.354 \\
\hline
\end{tabular}

suggests that the barycentric velocity has started increasing again, we can hypothesize that Cru-3AB has already covered approximately half of the orbit, in which case the orbital period of the binary $\mathrm{Cru}-3 \mathrm{AB}+\mathrm{Cru}-3 \mathrm{C}$ would be around 1500 days. Using this value as an initial guess for the orbital period, we find the orbital solution reported in Fig. 6 with the corresponding orbital parameters given in Table 7 . From Table 7 one can see that the barycentric velocity of the system $\mathrm{Cru}-3 \mathrm{AB}+\mathrm{Cru}-3 \mathrm{C}$ is quite consistent with the radial velocity of the other members of the Crux group, which suggests that the actual orbit might indeed be not very far from the one shown in Fig. 6. Assuming that Cru-3AB is composed by two solar-mass stars and using the mass function given in Table 7 , we estimate that the mass of Cru-3C might be about $0.5-0.6 M_{\odot}$. More data are obviously needed in order to check the validity of this preliminary orbit and improve the tertiary mass estimate made here. 


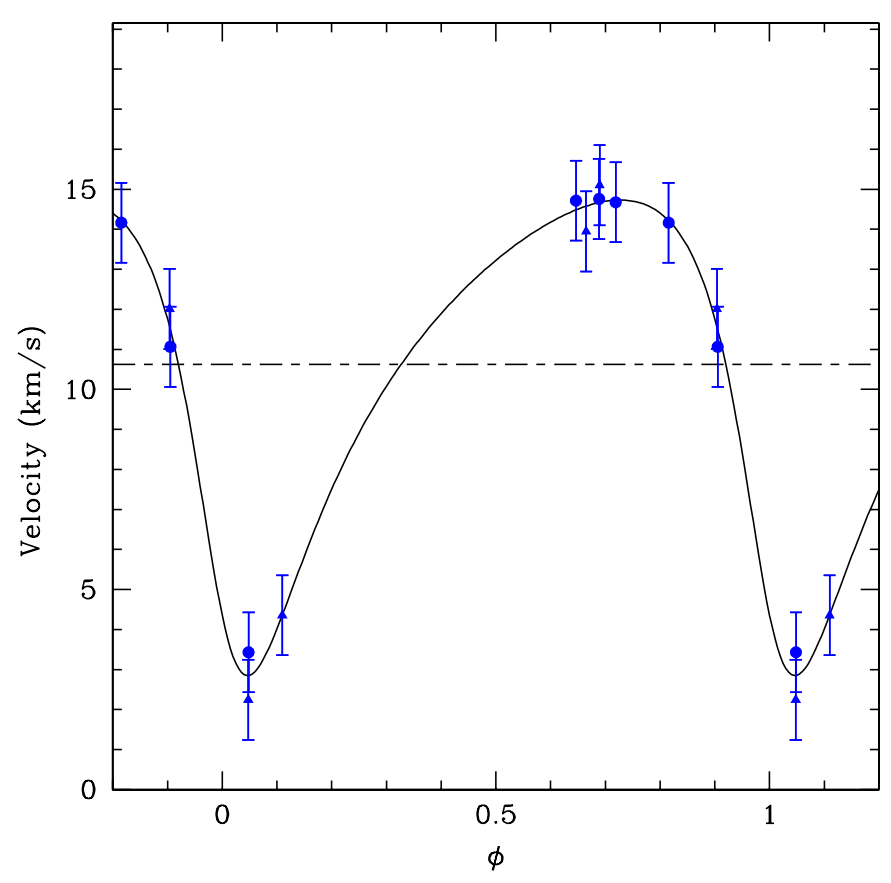

Fig. 6. Preliminary orbit of the center of mass of the spectroscopic binary. The error bars represent the standard deviation from the mean $R V$ in each observing period.

Table 6. Orbital parameters for the SB2 components of Cru-3

\begin{tabular}{lr}
\hline Parameter & Value/error \\
\hline \hline$P_{\text {orb }}(\mathrm{d})$ & $58.2748 \pm 0.0055$ \\
$T(\mathrm{HJD}-240000)^{a}$ & $51048.65 \pm 0.36$ \\
$e$ & $0.0675 \pm 0.0016$ \\
$\gamma_{\text {May } / 99}\left(\mathrm{~km} \mathrm{~s}^{-1}\right)$ & $14.722 \pm 0.032$ \\
$\omega(\mathrm{deg})$ & $34.61 \pm 1.93$ \\
$K_{1}\left(\mathrm{~km} \mathrm{~s}^{-1}\right)$ & $32.380 \pm 0.063$ \\
$K_{2}\left(\mathrm{~km} \mathrm{~s}^{-1}\right)$ & $33.955 \pm 0.063$ \\
$a_{1} \sin i(\mathrm{Gm})$ & $25.888 \pm 0.051$ \\
$a_{2} \sin i(\mathrm{Gm})$ & $27.147 \pm 0.051$ \\
$M_{2} / M_{1}$ & $0.954 \pm 0.004$ \\
$M_{1} \sin ^{3} i\left(M_{\odot}\right)$ & $0.8980 \pm 0.0038$ \\
$M_{2} \sin ^{3} i\left(M_{\odot}\right)$ & $0.8564 \pm 0.0037$ \\
No. of meas. $^{b}$ & $42(50)$ \\
$\mathrm{rms}_{1}\left(\mathrm{~km} \mathrm{~s}^{-1}\right)$ & 0.292 \\
$\mathrm{rms}_{2}\left(\mathrm{~km} \mathrm{~s}^{-1}\right)$ & 0.292 \\
Time span $($ days $)$ & 815 \\
\hline
\end{tabular}

Notes to Table: ${ }^{a}$ Time of passage to periastron. ${ }^{b}$ Number of measurements used for the orbital solution and, in parenthesis, total number of observations.

Statistically it appears that spectroscopic sub-systems are frequent in visual or wide spectroscopic binaries (Tokovinin \& Smekhov 2001). The fact that the eccentricity of the inner SB2 of Cru-3 is small (almost circular), and the outer is moderately high is consistent with recent results by Tokovinin \& Smekhov (2001). Also the "long" to "short" period ratio of about 29 for Cru-3 points towards dynamical stability of the triple: empirically, triples with period ratios $P_{\text {long }} / P_{\text {short }}>10$ are viewed upon as stable (Tokovinin 2000).
Table 7. Orbital parameters for the system Cru-3AB+Cru3C.

\begin{tabular}{ll}
\hline$P_{\text {orb }}($ days $)$ & 1688.15 \\
$T_{0}-2400000$ (HJD) & 50152.6914 \\
$e$ & 0.41 \\
$\gamma\left(\mathrm{km} \mathrm{s}^{-1}\right)$ & 10.616 \\
$K_{\mathrm{a}}\left(\mathrm{km} \mathrm{s}^{-1}\right)$ & 5.941 \\
$a_{\mathrm{a}} \sin i\left(10^{9} \mathrm{~m}\right)$ & 125.6742 \\
$f_{1}(m)\left(M_{\odot}\right)$ & $0.278 \times 10^{-1}$ \\
$\sigma(\mathrm{O}-\mathrm{C})\left(\mathrm{km} \mathrm{s}^{-1}\right)$ & 0.540 \\
Number of measur. & 11 \\
\hline
\end{tabular}

\section{Discussion}

The presence of the strong Li I $\lambda 6708$ absorption line in the spectra of stars later than G5 is a good indicator of youth, because Lithium is very efficiently destroyed by convective mixing in the stellar interiors when the temperature at the bottom of the convective layer reaches about $2 \times 10^{6}$ (Bodenheimer 1965; D'Antona \& Mazzitelli 1994). The capability offered by high-resolution spectroscopy to confirm the PMS nature of X-ray emitting PMS candidates, thourgh the use of Li equivalent width versus $\log T_{\text {eff }}$ diagram, has been shown in previous works (Magazzù et al. 1997; Covino et al. 1997; Wichmann et al. 1999; Alcalá et al. 2000).

With the exception of the star Cru-5, all the Crux stars do show the Li I $\lambda 6708$ absorption in their spectra, although the strength of the line in the stars Cru$2 \mathrm{E}$ and $\mathrm{Cru}-2 \mathrm{~W}$ is comparable to that of the nearby Ca I $\lambda 6717$ line, while in all the other stars the lithium line appears much stronger than $\mathrm{Ca} \mathrm{I}$.

Figure 7 shows the lithium equivalent width versus effective temperature for the stars in Cru. The upper envelopes for young open clusters adopted by Martín \& Magazzù (1998) and by Preibisch et al. (1998) are represented by the continuous and dotted lines, respectively. While the stars Cru-1, Cru-4, Cru- 6 and both components of the SB2 Cru-3 fall well above the upper envelope for ZAMS stars, the stars Cru-2E and Cru-2W fall below that boundary. Hence, Cru-2E and Cru-2W are most likely young ZAMS stars. On the other hand, Cru- 6 and the SB2 components of $\mathrm{Cru}-3$ lie close to the dividing line between the WTTS and PTTS regions and Cru- 1 and Cru- 4 fall on the PTTS area. Therefore, it is likely that all these objects are in the post-T Tauri phase.

When comparing the lithium abundance of these stars with that of stars in young clusters, like IC 2602 (see Fig. 8) it is evident that, except for Cru-2E and W and for Cru-5 (which lacks lithium), the other stars should be as young as, or younger than the IC 2602 stars, ie. younger than about 30 Myr.

The fact that the star $\beta$ Crux is a well established proper motion member of the Lower Centaurus-Crux subgroup of the Sco-Cen association, led FL97 to the conclusion that also the six X-ray selected Crux stars are actually members of the low-mass population of the Sco-Cen association. The mean radial velocity and distance of the 


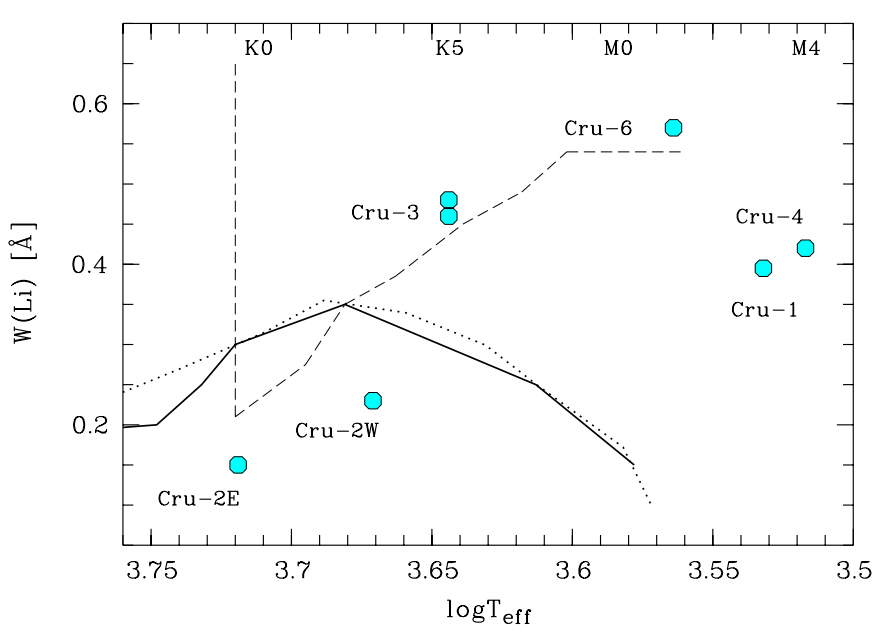

Fig. 7. Lithium equivalent width versus effective temperature. The thick and dotted lines represent the upper envelope for young open clusters as adopted by Martín \& Magazzù (1998) and Preibisch et al. (1998) respectively, while the dashed line indicates the WTTS and PTTS regions as described by Martín (1997).

Lower Centaurus-Crux group reported by de Zeeuw et al. (1999) are $+12 \mathrm{~km} \mathrm{~s}^{-1}$ and $118 \mathrm{pc}$ respectively. The radial velocity of $\beta$ Crux is $+15.6 \mathrm{~km} \mathrm{~s}^{-1}$ (Evans 1967). The radial velocity of Cru-1, Cru- 4 and $\mathrm{Cru}-6$, as well as the systemic radial velocity of $\mathrm{Cru}-3$ are consistent, within the errors, with the radial velocity of the Lower CentaurusCrux subgroup, while the radial velocities of $\mathrm{Cru}-2 \mathrm{E}$ and Cru-2W and Cru-5 are inconsistent. Therefore, the latter stars are very likely unrelated to the Sco-Cen association.

We can use the luminosities and effective temperatures reported in Table 3 to place the stars in the HR diagram. In Fig. 9 the position of the Crux stars in the HR diagram is compared with the theoretical pre-main sequence evolutionary tracks by Baraffe et al. (1998).

While the stars Cru-1, Cru-3, Cru-4 and Cru-6 fall well above the main sequence, approximately on the same isochrones with ages between 5 and 10 Myrs and masses between 0.3 and $1.2 M_{\odot}$, again the stars Cru-2E and $2 \mathrm{~W}$ and Cru-5 turn out to be unrelated to the other stars. Note also that Cru-5 has a high extinction which indicates that this object is probably a background K-type giant. Moreover, the X-ray - to - optical-flux $\left(f_{\mathrm{X}} / f_{\mathrm{V}}\right)$ ratio of $10^{-3.15}$, reported by PF96 for Cru-2, is in the range $-4.4<\log \left(f_{\mathrm{X}} / f_{\mathrm{V}}\right)<-2.8$ for $\mathrm{G}-\mathrm{K}$ type Pleiades stars (Stauffer et al. 1994), while the other Crux stars have higher $f_{\mathrm{X}} / f_{\mathrm{V}}$ ratios, which are more consistent with PMS stars. This gives further support to the conclusion that Cru-2E, Cru-2W and Cru-5 are not PMS stars. In addition, the projected rotational velocities of Cru-2E, Cru-2W and Cru-5 (see Table 2) are too low in comparison to those of low-mass PMS stars of similar spectral types.

Note that for the binary stars Cru-1 and Cru-3, represented with the circled dots in Fig. 9, the luminosities have been corrected as explained in Sect. 3.4. However, as pointed out in that section, it is difficult to determine

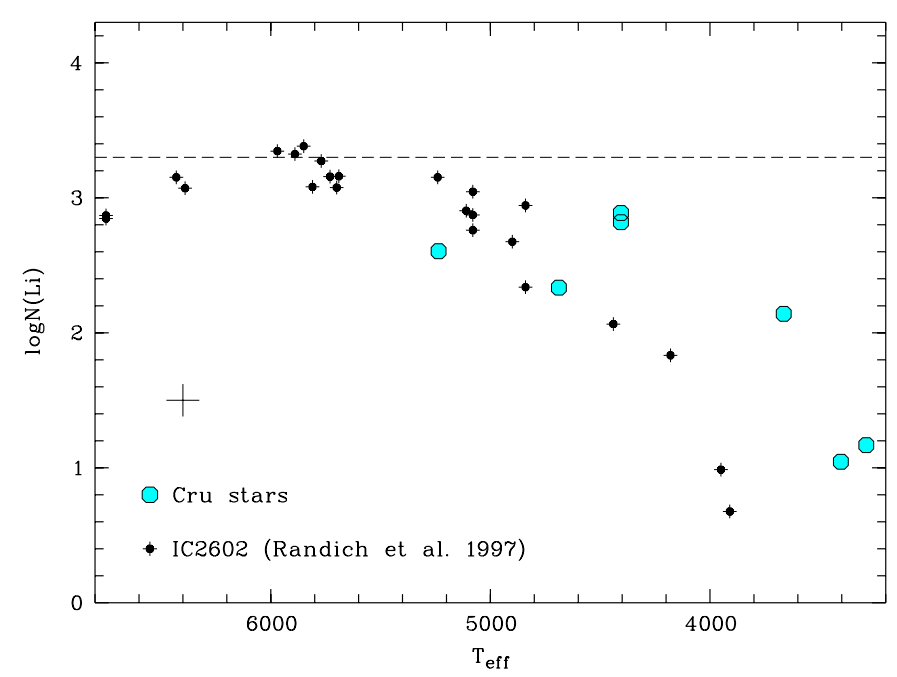

Fig. 8. Lithium abundance versus effective temperature for the Crux stars. The stars of the 30 Myr old cluster IC 2602 by Randich et al. (1997) are also plotted. The dashed line represents the cosmic lithium abundance of 3.3 in the $\log (H)=$ 12 scale.

the luminosity of the components of Cru- 1 and hence, it is not possible to separate its individual components in the HR diagram, unless some strong assumptions are made. For instance, that the luminosity ratio of the components is equal to the flux ratio in the $K$-band and also that the visual pair is indeed a physical binary, in which case the secondary visual component must be about one spectral class later than the primary because it emits 1.8 less flux (see Sect. 4.1). In this case, the age of the components of Cru-1 would fit quite well with that of Cru-3a and b, Cru-4 and Cru-6 (cf. Fig. 9).

Using the results on the masses derived from the HR diagram, one can speculate on what the orbital period of Cru- 1 would be if the projected separation of 0.25 arcsec is indicative of the mean separation. In this case, such separation would correspond to about $27 \mathrm{AU}$ at the distance of $110 \mathrm{pc}$; from the HR diagram, we derive upper and lower limits of $0.6 M_{\odot}$ and $0.38 M_{\odot}$ for the total mass of the system respectively. Using Kepler's third law, a period of about 200 years is estimated. We stress, however, that many assumptions have been made and that the mass and age estimates for the components, as well as for the orbital period determination of $\mathrm{Cru}-1$ have to be taken with care until more information regarding the colours of the secondary component will be available.

On the other hand, the dynamical mass of each one of the components of Cru-3ab is a factor of about 1.4 less than the mass inferred from the comparison with the theoretical tracks shown in Fig. 9. This means that, if those PMS tracks are correct, the inclination angle of the system is about $63^{\circ}$.

We conclude that the stars Cru-1, Cru-3, Cru- 4 and Cru-6 are indeed low-mass PMS, associated kinematically and coeval to the Lower Centaurus-Crux subgroup of the Sco-Cen association, and that the stars Cru-2 and Cru-5 are active stars unrelated to the association. 


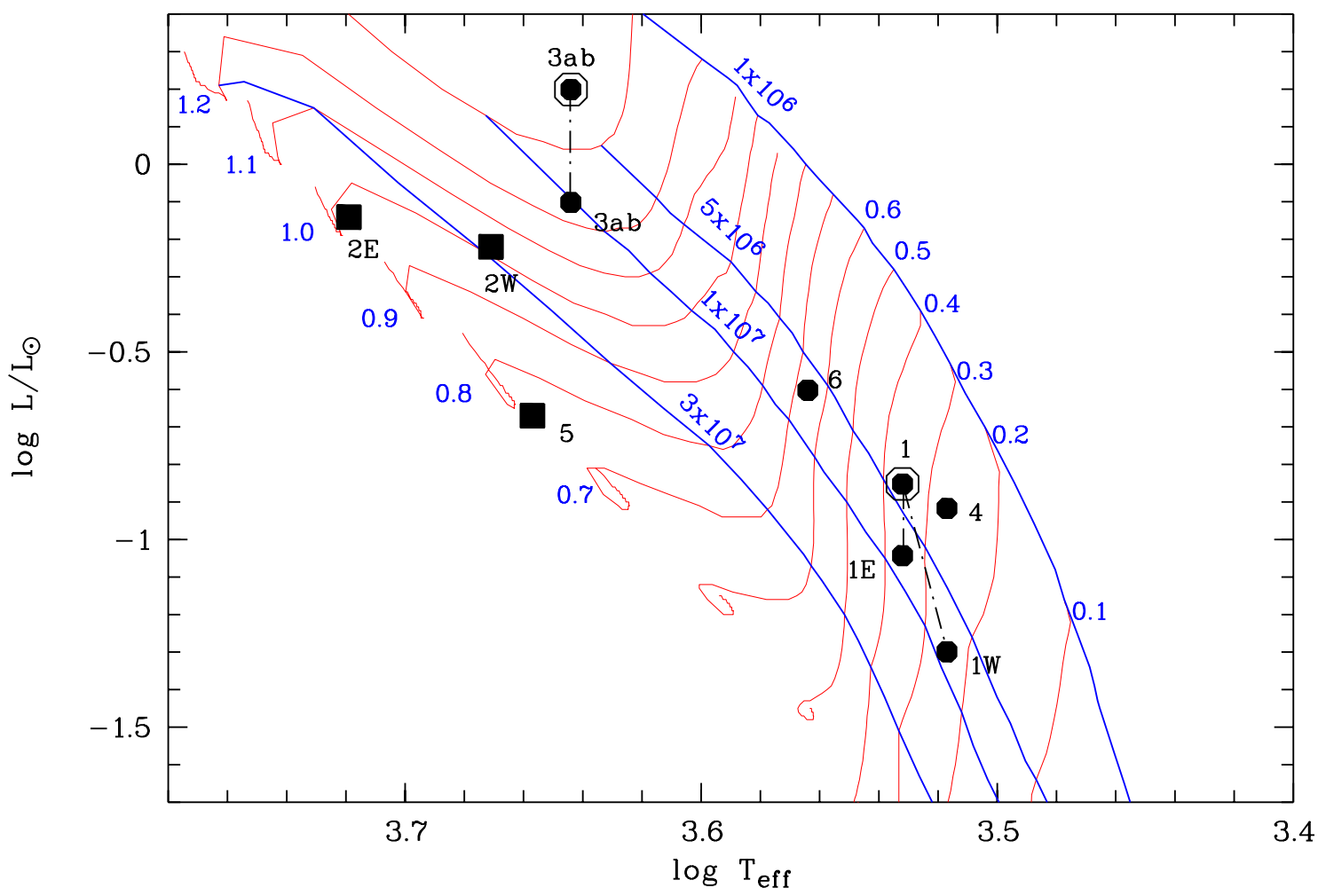

Fig. 9. Luminosity versus temperature diagram. The Crux stars are represented with the black dots and squares. The binary stars Cru-1 and Cru-3 are represented with the circled dots, while their individual components (connected with the dashed lines) are represented with dots. The theoretical pre-main sequence evolutionary tracks by Baraffe et al. (1998) (for $[\mathrm{M} / \mathrm{H}]=0$, $Y=0.282$ and $\left.\alpha=1 / H_{\mathrm{p}}=1.0\right)$ are overplotted.

Several other low-mass PMS stars have been found as counterparts of X-ray sources around high-mass stars that are, at the same time, part of an $\mathrm{OB}$ or $\mathrm{T}$ association. For instance, the RASS found several low-mass PMS stars spread around the Chamaeleon SFR (Alcalá et al. 1995, 1997), some of which were later confirmed to be associated with the B8-type star $\eta$ Cha, forming a cluster (Mamajek et al. 1999, 2000). Also Walter et al. (1998) found a small cluster of PMS stars around $\sigma$ Ori and Pozzo et al. (2000) identified many low-mass PMS star candidates as counterparts of ROSAT X-ray sources in the field around the Wolf-Rayet/O-type star $\gamma$ Velorum.

Since the velocity dispersion in star formation regions is of the order of a few $\mathrm{km} \mathrm{s}^{-1}$ (e.g. Lada \& Lada 1991), in a few $10^{7}$ years the small clusters are dissolved and are no longer recognized as such. Whether Cru-1, Cru-3, Cru- 4 and Cru- 6 are members of a small aggregate, in which $\beta$ Crux is the massive and central star, is not clear. The star $\beta$ Crux has a radial velocity of $+15.6 \mathrm{~km} \mathrm{~s}^{-1}$, which means a velocity dispersion relative to $\mathrm{Cru}-3$, Cru4 and Cru- 6 of about $3 \mathrm{~km} \mathrm{~s}^{-1}$ (note that the $R V$ of Cru-1, though more consistent with that of $\beta \mathrm{Cru}$, is variable). Assuming a distance of $110 \mathrm{pc}$, the studied stars would cover the observed spatial extent of about 1 degree in less than $1 \mathrm{Myr}$, moving with a velocity of $1-2 \mathrm{~km} \mathrm{~s}^{-1}$, which is inconsistent with the ages derived from the HR diagram. On the other hand, from their lithium content (see Fig. 8) we know that the Crux stars must be younger than 30 Myr. A 30 Myr population would expand some $30-60 \mathrm{pc}$, moving with a velocity of $1-2 \mathrm{~km} \mathrm{~s}^{-1}$. At a distance of $110 \mathrm{pc}$ such a population would appear completely dispersed. If the velocity dispersion is even higher, as it seems to be the case, the spread of the PMS stars would be even larger.

One possibility to explain the existence of a small aggregate would be that molecular material, which maintained the stars bounded during most of the cluster life, was recently cleared up by supernova winds in the same scenario as proposed by Mamajek et al. $(1999,2000)$ for the $\eta$ Cha cluster. However, the space density of the members of the hypothetical aggregate would be a factor of about three less than in the case of the $\eta$ Cha cluster.

The confirmed PMS stars and $\beta$ Crux do share, however, a common distance and age. Hence, they may be considered as a moving group in a more disperse population of PMS stars in Sco-Cen, rather than a small aggregate. In this case, many more X-ray emitting low-mass PMS stars are expected to be identified distributed on a large sky area as found in previous investigations of the RASS X-ray sources in other star forming complexes. The proper motion studies by de Zeeuw et al. (1999) revealed indeed a large number of objects with coherent proper motion in the Lower Centaurus-Crux subgroup. Therefore, we concur with the most plausible conclusion by FL97 that the stars detected in the PF96 ROSAT pointing, and confirmed here to be low-mass PMS stars, are just a few 
objects in a much larger loose group of low-mass PMS stars, which are members of the Lower Centaurus-Crux subgroup of the Sco-Cen association.

\section{Conclusions}

The high-resolution spectroscopic observations and the near IR photometry have allowed the confirmation of four low-mass PMS stars among the original sample proposed by PF96 and investigated by FL97 using low-resolution optical spectroscopy. These four stars have radial velocities consistent with those of the Lower Centaurus-Crux subgroup of the Sco-Cen association and hence are very likely part of the loose group of low-mass PMS stars of the Sco-Cen association. Many more widely spread X-ray emitting PMS stars are expected to be identified in the Lower Centaurus-Crux region. Given the high velocity dispersion and low spatial density of the Crux stars, the possibility that they form a small aggregate is unlikely. However, more X-ray observations with higher spatial resolution, extended to lower flux limits than those so far available will help to establish if there are more X-ray emitting PMS stars around $\beta$-Crux, and in that case, if they form a small cluster similar to the one around $\eta$ Cha.

Two of the stars (Cru-1 and Cru-3) are found to be a visual binary and a triple system, respectively. The number statistics is too low however for any conclusion regarding the binary fraction.

The PMS star Cru-3 has been found to be a hierarchical triple system in which one of the components is a SB2 itself. The separation of the hierarchical triple of about 4 AU, which means about 36 mas at the distance of $110 \mathrm{pc}$, as well as its orbital period of about 4.6 years, makes it a good candidate to be observed in the coming years with the Very Large Telescope Interferometer (VLTI), in order to fully solve the orbital elements, allowing the determination of the true mass of the components. The latter is crucial to put observational constraints on theoretical PMS evolutionary tracks, which are specially uncertain at the low-mass end.

Acknowledgements. We thank the referee Prof. W. A. Lawson for his comments. We thank the technical support by K. Brooks, A. Gonzalez, E. Matamoros, A. Sánchez and E. Wenderoth at the ESO $3.6 \mathrm{~m}$ telescope and by R. Vega, A. Torrejón, E. Pompei and P. Francois at the ESO $1.5 \mathrm{~m}$ telescope. This work has been financed by the Italian Ministero per la Ricerca Scientifica e Tecnologica.

\section{References}

Alcalá, J. M., Krautter, J., Schmitt, J. H. M. M., et al. 1995, A\&AS, 114, 109

Alcalá, J. M., Krautter, J., Covino, E., et al. 1997, A\&A, 319, 184

Baraffe, I., Chabrier, G., Allard, F., \& Hauschildt, P. H. 1998, A\&A, 337, 403

Bodenheimer, P. 1965, ApJ, 142, 451

Briceño, C., Hartmann, L. W., Stauffer, J. R., et al. 1997, AJ, 113,740
Covino, E., Alcalá, J. M., Allain, S., et al. 1997, A\&A, 328, 187

Covino, E., Catalano, S., Frasca, A., et al. 2000, A\&A, 361, L49

Covino, E., Melo, C. H. F., Alcalá, J. M., et al. 2001, A\&A, 375,130

de Jager, C., \& Nieuwenhuijzen, H. 1987, A\&A, 177, 217

de Zeeuw, P. T., et al. 1999, AJ, 117, 354

Feigelson, E. D. 1996, ApJ, 468, 306

Feigelson, E. D., \& Lawson, W. A. 1997, AJ, 113, 2130 (FL97)

Feigelson, E. D., \& Montmerle, T. 1999, ARA\&A, 37, 363

Favata, F., Micela, G., \& Sciortino, S. 1997, A\&A, 326, 647

Guillout, P., Sterzik, M. F., Schmitt, J. H. M. M., Motch, C., \& Neuhäuser, R. 1998, A\&A, 337, 113

Hunt, et al. 1998, AJ, 115, 2594

Lada, C. J., \& Lada, E. A. 1991, in The formation and evolution of star clusters, ed. K. Janes, 3

Magazzù, A., Martín, E. L., Sterzik, M. F., et al. 1997, A\&AS, 124,449

Mamajek, E. E., Lawson, W. A., \& Feigelson, E. D. 1999, ApJ, 516, L77

Mamajek, E. E., Lawson, W. A., \& Feigelson, E. D. 2000, ApJ, 544,356

Martín, E. L. 1997, A\&A, 321, 492

Martín, E. L. 1998, AJ, 115, 351

Martín, E. L., \& Magazzù, A. 1999, A\&A, 342, 173

Melo, C., Covino, E., Alcalá, J. M., \& Torres, G. 2001, A\&A, in press

Micela, G., Sciortino, S., \& Favata, F. 1993, ApJ, 414, 846

Park, S., \& Finley, J. P. 1996, AJ, 112, 693 (PF96)

Pavlenko, Y. V., \& Magazzù, A. 1996, A\&A, 311, 961

Perryman, M. A. C., Lindegren, L., Kovalevsky, J., et al. 1997, A\&A, 323, 49

Preibisch, T., Günther, E., Zinnecker, H., Sterzik, M., Frink, S., \& Röeser, S. 1998, A\&A, 333, 619

Pozzo, M., Jeffries, R. D., \& Naylor, T. 2000, MNRAS, 313, 23

Press, W. H., Teukolsky, S. A., Vetterling, W. T., \& Flannery, B. P. 1992, Numerical Recipes in Fortran. The Art of Scientific Computing, 2nd ed. (Cambridge: Cambridge Univ. Press), 650

Queloz, D. 1995, in IAU Symp. 167, ed. A. G. Davis Philip, 221

Randich, S., Aharpour, N., Pallavicini, R., Prosser, C. F., \& Stauffer, J. R. 1997, A\&A, 323, 86

Simkin, S. M. 1974, A\&A, 31, 129

Tokovinin, A. A., in The formation of binary stars, IAU Symp. 200, ed. H., Zinnecker, \& R. D., Mathieu, 84

Tokovinin, A. A., \& Smekhov, M. G. 2001, A\&A, submitted

Tripicchio, A., Severino, G., Covino, E., Terranegra, L., \& García López, R. J. 1997, A\&A, 327, 681

Tripicchio, A., Gomez, M. T., Severino, G., et al. 1999, A\&A, 345,915

Walter, F. M., ApJ, 306, 573

Walter, F. M., Alcalá, J. M., Neuhauser, R., Sterzik, M., \& Wolk, S. J. 2000, Protostars and Planets IV, ed. V. Mannings, A. P. Boss, \& S. S. Russell (University of Arizona Press), 273

Walter, F. M., Wolk, S. J., \& Sherry, W. 1998, ASP Conf. Ser., 154, The Tenth Cambridge Workshop on Cool Stars, Stellar Systems and the Sun, ed. R. A. Donahue, \& J. A. Bookbinder, 1793

Wichmann, R., Sterzik, M. F., Krautter, J., Metanomski, A., \& Voges, W. 1997, A\&A, 326, 211

Wichmann, R., Covino, E., Alcalá, J. M., Krautter, J., Allain, S., \& Hauschildt, P. H. 1999, MNRAS, 307, 909 